\title{
Cumulative Risk Estimation for Chemical Mixtures
}

\author{
M. Razzaghi
}

Bloomsburg University

\begin{abstract}
In reality, humans are always exposed to a combination of toxic substances and seldom to a single agent. Simultaneous exposure to a multitude of chemicals could result in unexpected consequences. The combined risk may lead to greater or less than a simple summation

of the effects induced by chemicals given individually. Here, a method is proposed for estimating the cumulative risk which is the risk associated with exposure to more than one chemical through different routes. The method is based on using the data to determine a suitable power transformation of the dose of each component of the mixture and fitting a dose-response model to the mixture under dose-addition. Necessary and sufficient conditions for the constancy of the relative potency between two chemicals in terms of the slopes of their corresponding dose-response models are derived and it is shown how the relative potency may be estimated directly from the joint dose-response model of the mixture. An example using a mixture of four chemicals is used for illustration.
\end{abstract}

Keywords. Box-Cox transformation; chemical mixtures; joint action; risk assessment.

\section{Introduction}

Concerns over health risks of individuals as the result of exposure to chemical compounds has been steadily growing especially over the last twenty years. 
Governments, regulatory agencies and international organizations have developed strategies for regulation of the use of chemicals for agricultural, commercial and industrial applications. Humans are generally exposed to mixtures of chemicals rather than a single substance. Such exposures occur through various environmental media and through multiple routes of exposure. In order to assess the joint toxicity of such complex exposures, it is necessary to develop methodologies that lead to scientifically creditable risk assessment of chemical mixtures. For this reason, several methods have been introduced in the last decade and the problem still attracts the attention of many scientists around the world since there is still no consensus on appropriate statistical methods for mixture risk assessment. Guidelines and approaches for health risks assessment of chemical mixtures have been described in a document developed by the US Environmental Protection Agency (US EPA, 2000). Accordingly, methods for risk assessment of chemical mixtures generally fall into two approaches. One approach is the whole mixture approach which usually involves direct evaluation of the mixture. That is, whenever possible, risk evaluation of the mixture is performed through assessment of health effects and exposure data on the whole mixture. This task can be accomplished by utilizing data directly on the mixture, data on a sufficiently similar mixture, or data on a group of similar mixtures. However, if data is not available on identical or a reasonably similar mixture, the alternative approach to risk assessment is based on the properties of individual components in the mixture. Using the component-based approach, recently Chen et al (2001) derived the dose-response function of the mixture for quantal response data and gave an estimate of the cumulative risk i.e. the risk for concurrent exposure for a group of chemicals that cause a common toxic effect. Their method utilizes the logarithmic transformation of the dose in the dose-response function. Although both untransformed dose and its logarithm are often used in dose-response assessments, it is shown by Razzaghi and Kodell (1992) that using the Box-Cox power transformation to obtain a suitable function of the dose often leads to an improved understanding of the joint action of the chemicals. Here, therefore, we consider the problem of cumulative risk estimation in a chemical mixture and derive the dose-response function of the mixture using the Box-Cox transformation. In section 2 we describe current approaches for component-based dose-response modeling of chemical mixtures and discuss the use of Box-Cox power transformation in section 3. Sections 4 and 5 are devoted to dose-response modeling of the mixture for the purpose of cumulative risk estimation and in section 6 our methodology is illustrated through an example. 


\section{Mixture Dose-Response Models}

Denote by $P(d)$ the probability of a toxic response to concentration $\mathrm{d}$ of a chemical. Assuming that $P(d)$ can be expressed by a suitable sigmoidal distribution function $\mathrm{F}$ such as the logistic or probit functions, the general approach to the analysis of quantal response data (Finney, 1978) is to assume that either

$$
P(d)=F(\alpha+\beta d)
$$

or

$$
P(d)=F(\alpha+\beta \log d)
$$

and apply a suitable linearizing transformation on $p(d)$ in order to obtain a linear response function in either the dose $d$ or its logarithm $\log d$. Regression methods are then used to estimate the parameters $\alpha$ and $\beta$.

Now, for mixtures of chemicals, the most widely used component-based methods fall under two categories of dose addition and response addition. According to the EPA (2000) document two chemicals are dose additive if one chemical is functionally a clone of the other and therefore the two chemicals behave similarly in terms of the primary physiological processes and hence act on the same biological site. Thus if $F_{1}(\cdot)$ and $F_{2}(\cdot)$ are respectively the doseresponse functions of the two chemicals, so that the probability of an adverse effect as the result of exposure to the does $d_{i}$ of chemical $i$ is $F_{i}\left(d_{i}\right)$ for $i=1,2$, then the probability of a toxic response to the combination of $d_{1}$ and $d_{2}$ may be expressed as

$$
\begin{aligned}
P\left(d_{1}, d_{2}\right) & =F_{1}\left(d_{1}+\rho d_{2}\right) \\
& =F_{2}\left(\frac{d_{1}}{\rho}+d_{2}\right)
\end{aligned}
$$

where $\rho$ is called the relative potency of chemical 2 to chemical 1 . Two chemicals are response additive, on the other hand, if they behave independently of one another, so that the body's response to the first chemical is the same whether or not the second chemical is present. Response addition, therefore, can be described by the statistical law of independent events. Thus the joint action of the two chemicals at doses $d_{1}$ and $d_{2}$ can be expressed as

$$
P\left(d_{1}, d_{2}\right)=F_{1}\left(d_{1}\right)+F_{2}\left(d_{2}\right)-F_{1}\left(d_{1}\right) \cdot F_{2}\left(d_{2}\right)
$$

Note that since cumulative risk assessment is based on chemicals sharing a common toxic effect arising from the same mechanism, dose addition is the more appropriate model for estimating the cumulative risk (Wilkinson et al, 2000). 
Kodell and Pounds (1991) applied equations (1) and (2) for the statistical analysis of the joint action of a mixture of two chemicals using the untransformed dose and its logarithmic transformation as suggested in Finney (1978). Razzaghi and Kodell (1992) generalized this approach by applying the Box-Cox power transformation to obtain the most suitable transformation determined by data. They show that their approach leads to a more refined characterization of the combined effect of two chemicals. Chen, et al. (2001) used dose addition along with the logarithm of doses to directly fit the combined dose-response function for any number of chemicals in the mixture. They then calculated the cumulative risk by estimating the relative potencies between chemicals from the joint dose-response function of the mixture through addition of the doses of individual compounds. Our goal, here is to generalize their approach and instead of the logarithmic transformation of the doses of all chemicals, apply the Box-Cox power transformation to determine the appropriate transformation of the doses.

\section{Box-Cox power transformation}

Consider the following family of dose-response models

$$
\begin{aligned}
P(d ; \alpha, \beta) & =F\left(\alpha+\beta \frac{d^{\lambda}-1}{\lambda}\right) \quad \lambda \neq 0 \\
& =F\{\alpha+\beta \log (d)\} \quad \lambda=0
\end{aligned}
$$

Here, the transformation on the dose is defined by a class of transformations indexed by the parameter $\lambda$. This class of transformations, suggested first by Box and $\operatorname{Cox}(1964)$ is a continuous function of $\lambda$ since $\lim _{\lambda \rightarrow 0}\left(\frac{d^{\lambda}-1}{\lambda}\right)=\log d$, and has the advantage of providing a more reliable model by allowing the data to choose the most appropriate transformation of the dose. The class includes logarithmic transformation more naturally in a larger and richer family of transformations. Now, for a mixture of two chemicals, if one chemical acts as if it is a simple dilution of the other, then the relative potency (dilution factor) between the two chemicals is a constant at every response level. In this case the effective dose of one chemical is simply a constant multiple of the effective dose of the other chemical. Thus if $d_{1}=\rho d_{2}$, then the dose- response of one chemical can be expressed in terms of the dose-response of the other chemical as

$$
P_{1}\left(d_{1}\right)=P_{2}\left(\frac{d_{1}}{\rho}\right)
$$


and

$$
P_{2}\left(d_{2}\right)=P_{1}\left(\rho d_{1}\right)
$$

where $P_{i}\left(d_{i}\right)=P\left(d_{i} ; \alpha_{i}, \beta_{i}\right)$ given by (3) for $i=1,2$. Then by using (3), we have

$$
\alpha_{1}+\beta_{1}\left(\frac{d_{1}^{\lambda}-1}{\lambda}\right)=\alpha_{2}+\beta_{2}\left\{\frac{\left(\frac{d_{1}}{\rho}\right)^{\lambda}-1}{\lambda}\right\}
$$

which is true for every $d_{1}$. Specifically, for $d_{1}=0$, we get

$$
\alpha_{1}=\alpha_{2}+\frac{\beta_{1}-\beta_{2}}{\lambda}
$$

and for $d_{1}=1$, we have

$$
\alpha_{1}=\alpha_{2}+\beta_{2}\left(\frac{1-\rho^{\lambda}}{\lambda \rho^{\lambda}}\right)
$$

leading to

$$
\rho=\left(\frac{\beta_{2}}{\beta_{1}}\right)^{\frac{1}{\lambda}}
$$

Note that we could similarly use (4) to obtain (5). Conversely, if slopes of the two dose- response functions are related according to (5), then for $P_{1}\left(d_{1}\right)=$ $P_{2}\left(d_{2}\right)$, we have

$$
\begin{aligned}
\alpha_{1}+\beta_{1}\left(\frac{d_{1}^{\lambda}-1}{\lambda}\right) & =\alpha_{2}+\beta_{1} \rho^{\lambda}\left(\frac{d_{2}^{\lambda}-1}{\lambda}\right) \\
& =\alpha_{2}+\beta_{1}\left(\frac{d_{1}}{d_{2}}\right)^{\lambda}\left(\frac{d_{2}^{\lambda}-1}{\lambda}\right)
\end{aligned}
$$

leading to

$$
\rho=\left\{1+\frac{\lambda\left(\alpha_{2}-\alpha_{1}\right)^{\frac{1}{2}}}{\beta_{1}}\right\}
$$

That is, the relative potency $\rho$ between the two chemicals is constant for all dose levels. Hence, we have proved that two chemicals have a constant relative potency if and only if the ratio of the slopes of their dose-response functions is a constant. This is a more general version of the theorem proved by Chen et al (2001) and as $\lambda \rightarrow 0$ their result is obtained.

If, on the other hand, the relative potency between the two chemicals is not a constant, so that the dilution factor of one chemical in terms of the other chemical varies at every response level, then the ratio of the slopes of their 
respective dose-response functions also varies at every response level. In this case, if $d_{1}=\delta\left(d_{2}\right)$ where $\delta(\cdot)$ is some appropriate function, then equal effective doses of $d_{1}$ for chemical 1 and $d_{2}$ for chemical 2 for which $P\left(d_{1}\right)=P_{2}\left(d_{2}\right)$ leads to

$$
\delta\left(d_{2}\right)=\left\{1+\lambda \frac{\left(\alpha_{2}-\alpha_{1}\right)}{\beta_{1}}+\frac{\beta_{2}}{\beta_{1}}\left(d_{2}^{\lambda}-1\right)\right\}^{\frac{1}{\lambda}}
$$

and a similar expression may be derived for equal effective dose of $d_{2}$ of chemical 2 in terms of $d_{1}$ of chemical 1 . Once again it is worth noting that

$$
\lim _{\lambda \rightarrow 0} \delta\left(d_{2}\right)=d_{2}^{\frac{\beta_{2}}{\beta_{1}}} \exp \left(\frac{\alpha_{2}-\alpha_{1}}{\beta_{1}}\right)
$$

which is the expression derived in Chen, et al (2001). Note also that in this case, the joint response from an exposure to dose $d_{1}$ of chemical 1 and dose $d_{2}$ of chemical 2 in terms of the dose-response of chemical 1 is given by

$$
P\left(d_{1}, d_{2}\right)=F\left[\alpha_{1}+\beta_{1} \frac{\left\{d_{1}+\delta\left(d_{2}\right)\right\}^{\lambda}-1}{\lambda}\right]
$$

which is not necessarily the same as the joint response obtained in terms of the dose-response of chemical 2.

\section{Mixtures with More than Two Chemicals}

Suppose now a compound consists of $\mathrm{k}$ chemicals. Suppose for the moment, that the relative potency between any two chemicals is also a constant. Let $\rho_{i j}$ be the relative potency (dilution factor) of chemical $j$ to chemical $i$ so that dose $d_{i}$ of chemical $i$ and dose $d_{j}$ of chemical $j$ are related by $d_{i}=\rho_{i j} d_{j}$ for $i, j=1 \cdots, k$ Clearly $\rho_{i j}=\frac{1}{\rho_{i i}}$ and $\rho_{i i}=1$. Now, the joint response of doses $d_{1}, d_{2}, \cdots, d_{k}$ of chemicals $1,2, \ldots, k$ respectively can be characterized in terms of the dose-response function of any of the $k$ chemicals and the probability of a toxic response is given by

$$
P\left(d_{1}, d_{2}, \ldots, d_{k}\right)=F\left\{\alpha_{i}+\beta_{i} \frac{\left(\mathbf{p}_{i}^{\prime} \mathbf{d}\right)^{\lambda}}{\lambda}\right\} \quad i=1, \ldots, k
$$

where $\rho^{\prime}=\left(\rho_{i 1}, \rho_{i 2}, \ldots, \rho_{i k}\right)$ and $\mathbf{d}^{\prime}=\left(d_{1}, d_{2}, \ldots, d_{k}\right)$.

In practice, one chemical in the mixture is selected as the so called "index" chemical and the probability of the toxic response is expressed in terms of that chemical. The choice of the index chemical depends on the availability of quantitative dose-response assessment of acceptable scientific quality. Normally, the index chemical will be the best studied component of the mixture 
with the largest body of acceptable scientific data. More specifically, if chemical $r, 1 \leqslant r \leqslant k$ is selected as the index chemical, then the probability of the toxic response is given by

$$
P\left(d_{1}, d_{2}, \ldots, d_{K}\right)=F\left\{\alpha_{r}+\beta_{r} \frac{\left(d_{r}+\sum_{j \neq r} \rho_{r j} d_{j}\right)^{\lambda}}{\lambda}\right\}
$$

from which, analogous to (6) we find that

$$
\rho_{r j}=\left\{1+\lambda \frac{\left(\alpha_{j}-\alpha_{r}\right)}{\beta_{r}}\right\}^{\frac{1}{\lambda}} j=1, \ldots, k_{j} j \neq r
$$

However, if the relative potency varies by response level, and if we define functions $\delta_{i j}(\cdot)$ to give the equal effective dose of $d_{i}$ of chemical i in terms of dose $d_{j}$ of chemical $j$ that is

$$
d_{i}=\delta_{i j}\left(d_{j}\right) \quad i, j=1, \ldots, k
$$

then an expression analogous to $(7)$ is obtained for $\delta_{i j}$. In practice, when the joint action of two chemicals can be characterized by dose additivity, then the assumption of a constant relative potency is often reasonable. However, if it is known that the relative potency changes at different response levels, then one can use a suitable mathematical function such as a polynomial to model the relative potencies.

\section{Cumulative Risk Assessment}

Although no single approach can be recommended for multiple chemical exposures, general guidelines are recommended in EPA (1986) and EPA (2000). According to these guidelines when dose additivity can be applied in the componentbased approach, then the toxicity of a chemical mixture is best determined by direct toxicologic evaluation. In other words, when individual studies are available for all of a mixture's components, the results may be used to develop a so called hazard index defined as

$$
\mathrm{HI}=\sum_{i=1}^{k} \frac{E_{i}}{A_{i}}
$$

where $E_{i}$ and $A_{i}$ are respectively the exposure level and the acceptable level of the ith chemical. However, because of experimental constraints e.g. cost, 
often such studies are not available on every component. If it can be assumed that the relative potency between any two chemicals in the compound is a constant then it suffices to have toxicologic evaluation of one of the chemicals (index chemical) in the mixture together with estimates of the constant relative potencies. Therefore, from (9) if $\hat{\alpha}, \hat{\beta}, \hat{\rho}_{r j}$ and $\hat{\lambda}$ are the estimates of the model parameters, then an estimate of the cumulative risk at the dose

$$
D=\hat{\rho}_{r 1} d_{1}+\hat{\rho}_{r 2} d_{2}+\cdots+\hat{\rho}_{r k} d_{k}
$$

of the mixture is given by

$$
P(D)=F\left(\hat{\alpha}_{r}+\frac{\hat{\beta}_{r} D^{\hat{\lambda}}}{\hat{\lambda}}\right)
$$

Equation (10) can equivalently be used to determine a safe exposure level (benchmark dose) for a given negligible value of risk. Therefore, if $R$ is the maximum acceptable level of risk then the corresponding exposure level, often used as the point of departure in risk assessment is given by

$$
\hat{D}^{*}=\left\{\hat{\lambda} \frac{F^{-1}(R)-\hat{\alpha}_{r}}{\hat{\beta}_{r}}\right\}^{\frac{1}{\lambda}}
$$

\section{Example}

To illustrate the methodology described in this paper, we use a data set of four analgesics given by Finney (1971, chapter 6, P.104). The experiment, first reported in Grewal, (1951) was designed to measure relative potencies of three toxicants relative to morphine. For completeness, we present the part of data that represent typical toxicological dose-response data in Table 1 . Chen et al (2001) used this data set to illustrate their method of cumulative risk estimation. Using a $\chi^{2}$ test, they also found that the hypothesis of constant relative potencies between the chemicals could not be rejected. Here, we adopt the proposed Box-Cox power transformation approach, together with the logistic dose-response function,

$$
P(d)=c+(1-c)\left\{\frac{\exp \left(\alpha+\beta \frac{d^{\lambda}-1}{\lambda}\right)}{1+\exp \left(\alpha+\beta \frac{d^{\lambda}-1}{\lambda}\right)}\right\}
$$

Without loss of generality, chemical 1 was taken as the index chemical. Using PROC NLIN in SAS (1999), the data set for the four chemicals was fitted to the model (9) with $r=1$ and the dose- response function given by (11). In 
order to estimate the transformation parameter $\lambda$, Draper and Smith (1998, chapter 13 P.280) suggests choosing a plausible interval around 0 and fitting the model with selected values of $\lambda$ from the interval. For each fit, the residual sum of squares is recorded and a graph of the residual sum of squares against $\lambda$ is plotted which gives the value of $\lambda$ that produces the smallest residual sum of squares. Figure 1 displays this graph, where we see that the minimum is attained at $\hat{\lambda}=0.09$. For this value of $\hat{\lambda}$, the parameter estimates with corresponding standard errors are given in Table 2. Hence, as in Chen et al (2001) if we are interested to estimate the cumulative risk at the exposure doses of $d_{10}=0.005, d_{20}=0.010, d_{30}=0.005$ and $d_{40}=.010$, then the equivalent total mixture dose is given by

$$
D=0.005+1.25 \times 0.010+3.59 \times 0.005+0.34 \times 0.010=0.03885
$$

from which the predicted cumulative risk from (11) is given by $1.42 \times 10^{-4}$. Using a logarithmic transformation, Chen et al obtained $1.47 \times 10^{-4}$ for the cumulative risk, showing a slight overestimation.

Alternatively, from (11), we find that for a given low acceptable risk $R$, an estimate of the corresponding safe exposure level is given by

$$
\hat{D}^{*}=\left\{\frac{\hat{\lambda}}{\hat{\beta}} \ln \left(\frac{R-c}{1-R}\right)+1-\frac{\hat{\alpha} \hat{\lambda}}{\hat{\beta}}\right\}^{\frac{1}{\hat{\lambda}}}
$$

and so, for example if $R=0.1$, an estimate of $E D_{10}$ is derived as $\hat{E} D_{10}=0.9764$ which can be used as the point of departure in order to estimate the reference dose.

\section{Conclusions}

In the absence of availability of data on the whole mixture, the process of risk assessment may be based on the toxic properties of the components in the mixture. The default approach for risk assessment of chemical mixtures using the component-based method utilizes the assumption that the total risk due to the mixture is simply the sum of the risk of individual components, provided that the risk associated with individual components of the mixture at the levels present in the mixture are low. By using dose addition and fitting a dose-response function to the total dose of a mixture of similar components, Chen et al (2001) showed that simply adding the risk estimates of components of the mixture may lead to an erroneous estimate of the risk for the mixture. Their approach utilizes the logarithmic transformation of the dose in the doseresponse function. Instead of using the logarithm as the default transformation 
of the doses of the components, here we have proposed a generalization of their approach through the incorporation of the Box-Cox power transformation. The Box-Cox transformation enables one to determine the most appropriate concentration transformation in a general approach to modeling and analysis of quantal response data. It is believed that by using the proposed approach, a more refined characterization of the cumulative risk may be obtained.

Note that in our approach, we took a common transformation parameter $\lambda$ in the Box-Cox model to obtain the transformed dose for each chemical in the mixture. It may be argued that ideally a different transformation parameter should be used for each component of the mixture. However, since our goal here is to obtain an estimate of the cumulative risk from similar chemicals, it makes sense to think that the optimal power transformation parameters for the components are close if not identical. In fact it is believed that the efficiency gained in the parameter estimation by reducing the size of the parameter space surpasses the additional accuracy obtained by taking different power transformation parameter for each component. Also, it is worth noting that in practice, not all components of the mixture may be similar. In that case it is often possible to break down the components of the mixture into subclasses where within each subclass the relative potency between any two chemicals is a constant. Similar to Chen, et al (2001), our methodology can be used to determine an estimate of the cumulative risk by using equation (8) to obtain an estimate of equal effective doses for classes with dissimilar chemicals.

Lastly, it ought to be emphasized that the procedure described in this paper only applies to mixtures with quantal responses i.e. when the toxic response of the subject is either presence or absence of the effect. Cumulative risk estimation for mixtures with quantitative responses was recently studied in Chen, et al. (2003), where the proposed methodology also used the logarithmic transformation of the dose. It may be useful to study the effect of using the Box-Cox transformation to determine the most suitable power transformation of the dose in the case of quantitative responses. That problem is currently under investigation and will be reported elsewhere.

\section{References}

Box, G.E.P.; Cox, D.R. (1964). An analysis transformation (with discussion) J. R. Stat. Soc. ser. B Stat. Methodol. 26, 211-252.

Box, G.E.P.; Cox, D.R. (1983). An analysis of transformations revisited, rebutted. J. Amer. Statists. Assoc., 77, 209-210. 
Chen, J.J.; Chen, Y.J.; Rice, G.; Teuscher, L.K.; Hamernik, K.; Protzel, A.; Kodell, R.L. (2001). Using dose addition to estimate cumulative risks from exposures to multiple chemicals. Regul. Toxicol. Pharm. 34,35-41.

Chen, J.J.; Chen, Y.J.; Teuschler, L.K.; Rice, G.; Hamernik, K.; Protzel, A,; Kodell, R.L. (2003). Cumulative risk assessment for quantitative response data Environmetrics 14, 339353

Draper, N.R.; Smith, H. (1998). Applied Regression Analysis 3th ed. Wiley, New York.

EPA (1986). Guidelines for Health Risk from Exposure to Chemical Mixtures. U.S. Environmental Protectopn Agency. Fed. Reg. 51, 34014.

EPA (2000). Supplementary Guidance for Conducting Health Risk Assement of Chemical Mixtures. U.S. Environmental Protecion Agency Risk Assessment Forum, Washington, D.C.

Finney, D.J. (1971). Probit Analysis, 3th ed. Cambridge University Press, Cambridge.

Finney, D.J. (1978). Statistical Methodisin Biological Assay, 3th ed. Griffin, London.

Grewel, R.S. (1952). A method for testing analgesics in mice. Br. J. Pharm. Chemother. 7, 433-37.

Kodell, R.L.; Pounds, J.G.(1991). Assessing the toxicity of mixtures of chemicals. Statistics in Toxicology, Krewski, D.; Franklin, C. (eds.). Gordon Breach, New York.

Razzaghi, M.; Kodell, R.L. (1992). Box-Cox transformation in the analysis of combined effects of mixtures of chemicals. Environmetrics 3, 319-334.

Wilkinson, C.F.; Christoph, G.R.; Julien, E.; Kelly, J.M.; Kronenberg, J.; McCarthy, J.; Reiss, R. (2000). Assessing the risks of exposures to multiple chemicals with a common mechanism of toxicity: How to cumulate? Regul. Toxicol. Pharm. 31, 30-43. 


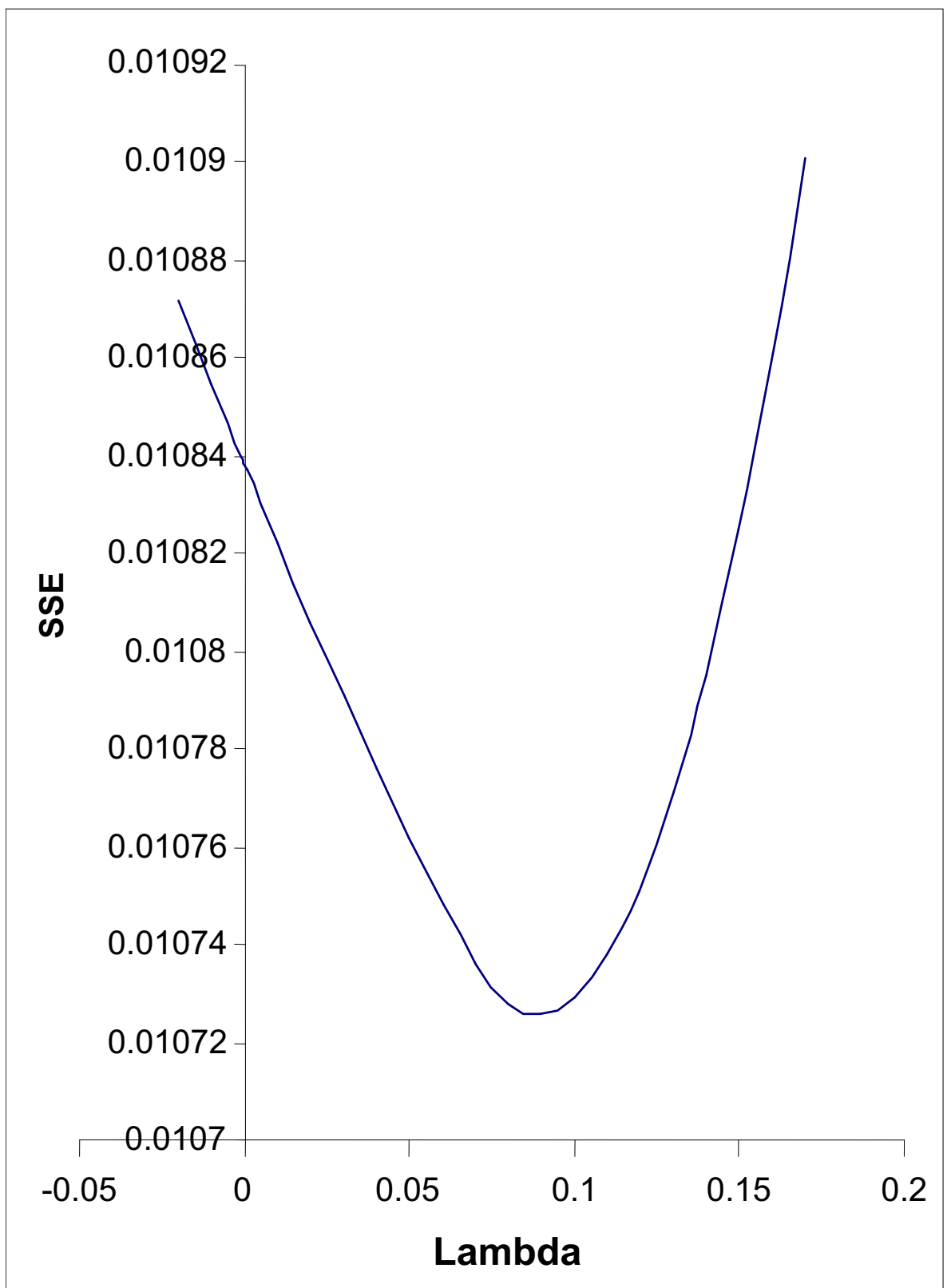

Graph 1. Error sum of squares aginst varying plausible values of the Box-Cox transformation parameter $\lambda$. 
Table 1. Percent of mice responding to varying doses of four chemicals.

\begin{tabular}{l|cccc}
\hline \hline \multirow{3}{*}{ Morphine } & Dose $\left(\frac{\mathrm{mg}}{\ell}\right)$ & $\begin{array}{c}\text { Number } \\
\text { of Animals }\end{array}$ & $\begin{array}{c}\text { Number } \\
\text { of Responses }\end{array}$ & $\begin{array}{c}\text { Percent } \\
\text { Responses }\end{array}$ \\
& 1.50 & 103 & 19 & 18 \\
& 3.00 & 120 & 53 & 44 \\
Amidone & 6.00 & 123 & 83 & 67 \\
& 1.50 & 60 & 14 & 23 \\
& 3.00 & 110 & 54 & 49 \\
& 6.00 & 100 & 81 & 81 \\
& & & & 34 \\
Phenadoxone & 0.75 & 90 & 31 & 68 \\
& 1.50 & 80 & 54 & 89 \\
& 3.00 & 90 & 80 & 22 \\
& & & & 32 \\
& 5.00 & 60 & 13 & 53 \\
& 7.50 & 85 & 27 & 61 \\
& 10.00 & 60 & 32 & 73 \\
\hline
\end{tabular}

Table 2. Maximum likelihood estimates of model parameter for the optimal transformation parameter $\lambda=0.09$.

\begin{tabular}{l|cccccc}
\hline \hline Parameter & $\mathrm{c}$ & $\alpha$ & $\beta$ & $\rho_{12}$ & $\rho_{13}$ & $\rho_{14}$ \\
\hline MLE & $10^{-8}$ & -2.16 & 1.56 & 1.25 & 3.59 & 0.34 \\
$\mathrm{SE}$ & 0.00 & 0.145 & 0.087 & 0.154 & 0.454 & 0.037 \\
\hline
\end{tabular}

| Received: May 2, 2005

\section{Mehdi Razzaghi}

Computer Science and Statistics,

Department of Mathematics,

Bloomsburg University,

Bloomsburg.

e-mail: Razzaghi@bloomu.edu 